\title{
The effect of antenatal corticosteroids on maternal serum glucose in women with diabetes
}

\author{
Allison Kreiner ${ }^{1}$, Karen Gil ${ }^{1,2}$, Justin Lavin ${ }^{1,2^{*}}$ \\ ${ }^{1}$ Department of Obstetrics and Gynecology, Akron General Medical Center, Akron, Ohio, USA \\ ${ }^{2}$ Northeast Ohio Medical University, Rootstown, Ohio, USA \\ Email: akstork@yahoo.com
}

Received 27 February 2012; revised 23 March 2012; accepted 2 April 2012

\begin{abstract}
Objective: To evaluate changes in serum glucose values following administration of corticosteroids in women with gestational (GDM) or pre-gestational diabetes (PGDM). Method: Patients with diagnoses of pregnancy and GDM or PGDM from 2006-2009 who received corticosteroids for enhancement of fetal pulmonary maturity as an inpatient were identified following IRB approval. Fasting (FSG) and two hour post prandial serum (PPSG) glucose levels, and oral anti-hyperglycemic agents and insulin given were recorded. Day 1 (D1) was the day the first dose of corticosteroid was administered. The percentage of women with FSG > $95 \mathrm{mg} / \mathrm{dL}$ and the percentage with at least one PPSG $>120 \mathrm{mg} / \mathrm{dL}$ during each day were calculated. Data were analyzed utilizing SPSS v15. Results: Fifty-five patients met the study criteria. FSG was greater than $95 \mathrm{mg} / \mathrm{dL}$ in over $90 \%$ of women on Days 2 and 3 and remained elevated in $51 \%$ on Day 4. At least one PPSG was greater than $120 \mathrm{mg} / \mathrm{dL}$ in $81 \%-98 \%$ on Days $1-3$, and in over $60 \%$ on Days 4 - 6. The majority of women taking insulin received increases in doses that were less than double their regular dose (26 out of 33 ); the others received higher doses. Three of 6 patients who began the study taking glyburide were started on insulin. Of the 19 who began the study with diet controlled GDM, 11 were started on medication. Conclusion: Women with GDM or PGDM who are administered antenatal corticosteroids will experience prolonged serum glucose levels above the range associated with optimal pregnancy outcome.
\end{abstract}

Keywords: Gestational Diabetes; Fetal Lung Maturity; Glucose Regulation

\section{INTRODUCTION}

Several studies have demonstrated that the administra-

${ }^{*}$ Corresponding author. tion of corticosteroids for the enhancement of fetal pulmonary maturity results in an increase in maternal glucose levels [1-5]. Very few studies, however, have examined the effect of administration of corticosteroids for the enhancement of fetal pulmonary maturity in women with gestational (GDM) or pregestational (PGDM) diabetes [6,7]. Mathiesen et al. examined the effect of increasing the dose of insulin given during betamethosone treatment in insulin dependent pregnant women [6]. They found that it was possible to prevent severe hyperglycemia by increasing the subcutaneous insulin dose by approximately $40 \%$ shortly after the glucocorticoid injection, before the rise in blood glucose levels. There remained a great deal of variability in women's responses, however, and median blood glucose values were still higher than optimal. Real-time continuous glucose monitoring and provision of intravenous insulin, in pregnant diabetic women may be an option for controlling glucose levels during betamethasone administration [7], however, this technique is not available in most non-research clinical practice settings. Currently, the management of pregnant diabetic patients admitted for corticosteroid administration for the enhancement of fetal lung maturity rests with the individual practitioners utilizing subcutaneously administered insulin.

Given the known effects of corticosteroids on blood glucose levels, and the variability in response among diabetic women, dosages of insulin are adjusted on an individual basis in clinical practice. This case series was conducted in a university affiliated community based hospital. The study was designed to determine if the management of women on an individual basis provided reasonable blood glucose control during the days following administration of corticosteroids.

\section{MATERIALS AND METHODS}

Using the hospital's computerized quality assurance program, charts of patients with the diagnoses of pregnancy and either GDM or PGDM from the years 2006-2009 were identified following IRB approval. From this group, 
all women who received corticosteroids for the enhancement of fetal pulmonary maturity during an inpatient admission were selected. Data recorded included: maternal age, gestational age, White classification, indication for steroids, fasting (FSG) and two hour post prandial serum (PPSG) glucose levels, height and weight. Doses of oral anti-hyperglycemic agents and insulin given were recorded. Data collection began the day before steroids were given and continued until discharge or delivery.

The day the first dose of corticosteroid was administered was designated Day 1 (D1). Steroids were given on D1 and D2. The percentage of women with FSG $>95$ $\mathrm{mg} / \mathrm{dL}$ and the percentage of women who had at least one PPSG $>120 \mathrm{mg} / \mathrm{dL}$ during each day were also calculated. Body mass index (BMI) was calculated. Chi square analyses were performed utilizing SPSS v15.

\section{RESULTS}

Fifty-five patients met the study criteria: 19 with $\mathrm{GDMA}_{1}$, 11 of whom advanced to $\mathrm{GDMA}_{2}$ after corticosteroid therapy, and 17, 13, 4, and 2 women who had $\mathrm{GDMA}_{2}$, Class B, Class C, and Class D diabetes on admission, respectively. Mean gestational age at admission was 30.9 \pm 0.3 weeks (SEM, range 23 - 34 weeks). Indications leading to admission and steroid administration represent relatively common complications of pregnancy, with the exception of the umbilical cord complications (Table 1). Historical data provided on admission indicated that most women were in good control i.e. below target levels of $95 \mathrm{mg} / \mathrm{dL}$ and $120 \mathrm{mg} / \mathrm{dL}$ for FSG and PPSG, respectively, prior to hospitalization.

FSG and PPSG were not available for all women at all time points. Data are presented for Days 1 - 6 as sample size continued to decrease due to women being discharged and/or delivered. FSG was greater than 95 $\mathrm{mg} / \mathrm{dL}$ in over $90 \%$ of all women on Days 2 and 3 and remained elevated in $51 \%$ on Day 4 (Figure 1). At least one PPSG was greater than $120 \mathrm{mg} / \mathrm{dL}$ in $81 \%-98 \%$ of all women on Days $1-3$, and in over $60 \%$ of women on Days 4 - 6 (Figure 1). There were no significant differences in the percent of women with GDM and PGDM who had elevated FSG on Days 1 - 3 (Table 2); on Day 4 a significantly higher percentage of women with PGDM had elevated FSG $(p=0.048)$. There were no significant differences in the proportion of women with GDM and PGDM who had at least one elevated PPSG.

Mean percent changes in insulin dose were analyzed for all women in the study who took insulin. Two patients who received a $2200 \%$ and $1400 \%$ increase in insulin doses, respectively, were eliminated from further evaluation because it was thought that their increases were so extreme that their inclusion would distort the
Table 1. Indication for corticosteroid administration to promote fetal lung maturity.

\begin{tabular}{ccc}
\hline Indication & Number & Percentage \\
\hline Preterm Labor & 18 & $32.7 \%$ \\
Non Reassuring Fetal Testing & 7 & $16.4 \%$ \\
PPROM & 9 & $12.7 \%$ \\
Pre-Eclampsia & 6 & $10.9 \%$ \\
Oligohydramnios & 4 & $7.3 \%$ \\
Placental Abruption & 3 & $5.5 \%$ \\
Chronic Hypertension with & 3 & $5.5 \%$ \\
Super-Imposed Pre-Eclampsia & 2 & $3.6 \%$ \\
Intrauterine Growth Restriction & 1 & $1.8 \%$ \\
Severe Pre-Eclampsia & 1 & $1.8 \%$ \\
Umbilical Cord Varicocele & 1 & $1.8 \%$ \\
Dilated Umbilical Vein & &
\end{tabular}

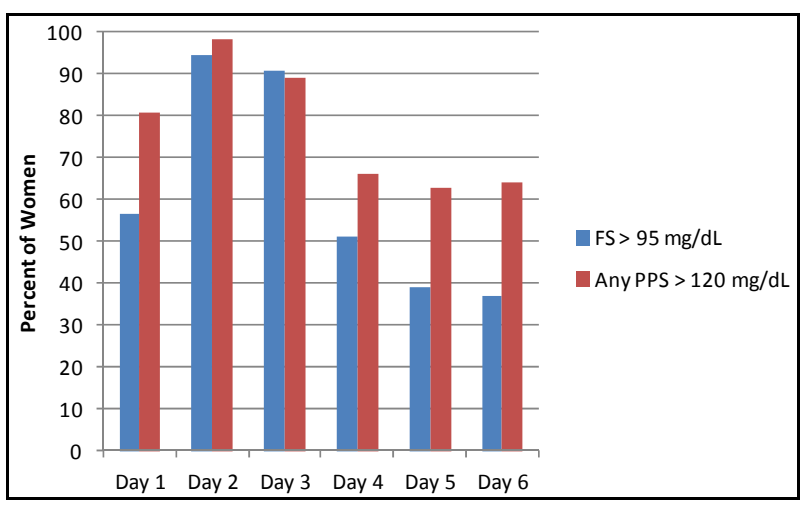

Figure 1. Percentage of women with fasting blood glucose (FSG) greater than $95 \mathrm{mg} / \mathrm{dL}$ and at least one two hour post prandial blood glucose (PPSG) greater than $120 \mathrm{mg} / \mathrm{dL}$ (sample sizes for FSG on Days 1 - 6 were 23, 54, 54, 51, 46, 38; sample sizes for PPSG on Days 1 - 6 were 52, 54, 54, 49, 46, 36).

data. Among the remaining patients, there appeared to be a bimodal distribution in dosage changes. Five patients received changes in their insulin regimen that on at least one day more than doubled their original insulin dose (range of $125 \%$ to $300 \%$ ). For these women the mean percent changes in insulin dose on D2 - D6 relative to doses given on Day 1 were $137 \%(\mathrm{n}=5), 217 \%(\mathrm{n}=5)$, $233 \%(\mathrm{n}=4), 276 \%(\mathrm{n}=2)$, and $225 \%(\mathrm{n}=2)$. The remaining 26 women required doses of insulin that were less than double their preadmission dose. The mean percent changes in insulin dose on D2 - D6 relative to doses given on Day 1 for these patients were $15.5 \%(\mathrm{n}=24)$, $16.3 \%(n=22), 4.5 \%(n=20), 11.8 \%(n=16)$, and $8.4 \%$ $(\mathrm{n}=14)$.

Six patients began the study taking glyburide and 3 
Table 2. Percent of women with fasting (FSG) or at least one post-prandial (PPSG) blood glucose above target levels.

\begin{tabular}{|c|c|c|c|c|}
\hline & $\begin{array}{l}\text { Class A1 and A2 } \\
\mathrm{FSG}>95 \mathrm{mg} / \mathrm{dL}\end{array}$ & $\begin{array}{c}\text { Class B, C, D } \\
\text { FSG }>95 \mathrm{mg} / \mathrm{dL}\end{array}$ & $\begin{array}{c}\text { Class A1 and A2 } \\
\text { PPSG }>120 \mathrm{mg} / \mathrm{dL}\end{array}$ & $\begin{array}{c}\text { Class B, C, D } \\
\text { PPSG }>120 \mathrm{mg} / \mathrm{dL}\end{array}$ \\
\hline Day 1 Steroids & $53 \%(\mathrm{n}=15)$ & $63 \%(\mathrm{n}=8)$ & $76 \%(n=33)$ & $90 \%(\mathrm{n}=19)$ \\
\hline Day 2 Steroids & $91 \%(\mathrm{n}=35)$ & $100 \%(\mathrm{n}=19)$ & $97 \%(\mathrm{n}=35)$ & $100 \%(\mathrm{n}=19)$ \\
\hline Day 3 & $94 \%(n=36)$ & $83 \%(n=18)$ & $89 \%(n=36)$ & $89 \%(n=18)$ \\
\hline Day 4 & $41 \%(\mathrm{n}=34)$ & $71 \%(\mathrm{n}=17)^{*}$ & $69 \%(\mathrm{n}=32)$ & $53 \%(17)$ \\
\hline Day 5 & $43 \%(n=30)$ & $31 \%(n=16)$ & $67 \%(n=30)$ & $56 \%(16)$ \\
\hline Day 6 & $33 \%(\mathrm{n}=24)$ & $43 \%(n=14)$ & $70 \%(n=23)$ & $54 \%(13)$ \\
\hline
\end{tabular}

${ }^{*} \mathrm{p}=0.048$, Class B, C, D versus Class A1, A2.

were started on insulin in addition to glyburide after corticosteroid administration. One of those 3 individuals also underwent an increase in glyburide dosage from 5.0 $\mathrm{mg}$ twice daily to $7.5 \mathrm{mg}$ twice daily. Three women with $\mathrm{GDMA}_{2}$ were taking $1500 \mathrm{mg}$ daily of metformin and their dosage did not change after corticosteroid administration.

Of the 19 patients who began the study with diet controlled GDM $\left(\mathrm{GDMA}_{1}\right), 11$ experienced serum glucose levels deemed sufficiently high to require the initiation of medication to improve glycemic control. Eight women were started on glyburide and 3 were started on insulin. Among these 11 women, 7 (63.6\%) were no longer on medication, and $4(36.4 \%)$ remained on some type of antihyperglycemic agent at the time of delivery.

The relationship between insulin dosage change and BMI was analyzed for the three groups. Group 1 included the 2 women whose insulin doses increased by more than $1000 \%$, group 2 consisted of the 5 women whose insulin doses more than doubled, and group 3 included 26 patients whose insulin doses less than doubled. The BMI of the two women in Group 1 were 39 and 47. There was no significant difference between mean BMI of women whose insulin dose more versus less than doubled ( $32.9 \pm 3.6$, range: $22-44 ; 33.85 \pm 2.0$, range: $19-53$, respectively). Within the group of 26 women whose dose less than doubled, the correlation between percent change in insulin dose on Day 2 and BMI approached significance $(r=-0.348, p=0.096)$.

Control of blood glucose on the day of delivery or discharge was examined in women who were delivered or discharged within 6 days $(\mathrm{n}=22)$ versus women who were in the hospital for longer than 6 days $(n=33)$. A nonsignificantly greater percentage of women who delivered or were discharged earlier versus later had FSG greater than $95 \mathrm{mg} / \mathrm{dL}$ on the day of delivery or discharge $\left(59 \%\right.$ versus $\left.34 \%, \chi^{2}=3.225, \mathrm{p}=0.073\right)$. There was no significant difference in the proportion of women who delivered or were discharged early versus later who had at least one PPSG greater than $120 \mathrm{md} / \mathrm{dL}$ on the day of discharge or delivery ( $41 \%$ versus $30 \%)$.

\section{DISCUSSION}

The 1994 NIH Consensus Conference on antenatal corticosteroids and the American Congress of Obstetricians and Gynecologists have both recommended that women at risk to deliver infants at gestational ages of 24 to 34 weeks be treated with corticosteroids in an attempt to accelerate fetal pulmonary maturity $[8,9]$. The risk of preterm delivery is increased in women with PGDM [10], and the prevalence of PGDM is increasing [11]. Thus it is highly likely that physicians who care for pregnant women will encounter patients with diabetes receiving corticosteroids to enhance fetal pulmonary maturity.

The data generated in this study suggest virtually all women with PGDM or GDM will experience deterioration in their glycemic control resulting in serum glucose levels above the range associated with optimal pregnancy outcome despite ongoing care and increases in insulin administration. Previous studies in non-diabetic women, have suggested that the impact of corticosteroids on maternal blood glucose lasts 24 hours [4], although alterations in a one hour glucose tolerance test were seen three days following betamethasone administration [3]. However the data generated in this study demonstrate that in a large proportion of diabetic patients, despite efforts by clinicians with substantial experience caring for women with diabetes, suboptimal glucose control may continue for at longer period of time. Of the women who were in the hospital longer than 6 days, 34\% had suboptimal FSG and 30\% had suboptimal PPSG at the time of discharge or delivery.

The findings reported here support the concept of increasing the dose of insulin delivered earlier [6]. In designing future studies, the $16 \%$ average increase in insulin dosage observed on Days 2 and 3 for those women whose insulin dosages less than doubled suggests that a relatively modest increase, if given early, may be sufficient to maintain glycemic control for most diabetic 
women. Increasing the dose of insulin earlier i.e. when the steroids are administered as opposed to waiting for glucose levels to deteriorate, may prevent the increase in serum glucose levels seen in virtually all women on Days 2 and 3. However, for a small subset of women even beginning the increase in insulin early may not be sufficient; they may require substantially more insulin. No clearcut relationships were observed between BMI and insulin requirements in this preliminary analysis. However, future research should work to identify factors that affect amount of insulin required to maintain euglycemia. Until these factors are identified, there may be subsets of women whose glycemic response will be extreme.

Approximately half of women with diet controlled gestational diabetes required the initiation of pharmacologic therapy to maintain glycemic control. Within this group of women, a large minority (36\%) required continued antihyperglycemic medication until delivery. Thus, the administration of corticosteroids may have long term hyperglycemic effects in women with previously diet controlled gestational diabetes and future studies should be designed to evaluate this effect in grater detail.

Limitations of the current study include it being a single institution retrospective study. However, the effects obtained were large and appear to warrant closer investigation. A prospective study is currently being designed which will further investigate this problem that is likely to be increasingly prevalent.

\section{REFERENCES}

[1] Landy, J.H., Isada, N., McGinnis, J., Ratner, R. and Grossman III, J.H. (1988) The effect of chronic steroid therapy on glucose tolerance in pregnancy. American Journal of Obstetrics \& Gynecology, 159, 612-615.

[2] Fisher, J., Smith, R., Lagrandeur, R. and Lorenz, R. (1997) Gestational diabetes mellitus in women receiving betaadrenergics and corticosteroids for threatened preterm delivery. Obstetrics \& Gynecology, 90, 880-883. doi:10.1016/S0029-7844(97)00544-9

[3] Mastrobattista, J.M., Patel, N. and Monga, M. (2001)
Betamethasone alteration of the one-hour glucose challenge test in pregnancy. The Journal of Reproductive Medicine, 46, 83-86.

[4] Foglia, L., Deering, S., Lim, E. and Landy, H. (2008) Maternal glucose levels after dexamethasone for fetal lung development in twin vs singleton pregnancies. American Journal of Obstetrics \& Gynecology, 199, 380-381. doi:10.1016/j.ajog.2008.08.004

[5] Star, H.J., Sosa, M.E. and Carpenter, M.W. (2000) Glucocorticoid-associated maternal hyperglycemia: A randomized trial of insulin prophylaxis. Journal of MaternFetal Medicine, 9, 273-277. doi:10.1002/1520-6661(200009/10)9:5<273::AID-MFM3 $>3.0 . \mathrm{CO} ; 2-\mathrm{N}$

[6] Mathiesen, E.R., Christensen, A.B., Hellmuth, E., Hornnes, P., Stage, E. and Damm, P. (2002) Insulin dose during glucocorticoid treatment for fetal lung maturation in diabetic pregnancy: Test of an algorithm [correction of analgoritm]. Acta Obstetricia et Gynecologica Scandinavica, 81, 835-839.

doi:10.1034/j.1600-0412.2002.810906.x

[7] Iafusco, D., Stoppoloni, F., Salvia, G., Vernetti, G., Passaro, P., Petrovski, G. and Prisco, F. (2008) Use of real time continuous glucose monitoring and intravenous insulin in type 1 diabetic mothers to prevent respiratory distress and hypoglycaemia in infants. BMC Pregnancy and Childbirth, 8, 23. doi:10.1186/1471-2393-8-23

[8] National Institutes of Health (NIH) (1994) Effect of corticosteroids for fetal maturation on perinatal outcomes. NIH Consensus Statement, 12, 1-24.

[9] American College of Obstetricians and Gynecologists (ACOG) (2001) Clinical management guidelines for obstetrician-gynecologists. ACOG Practice Bulletin No. 30. Obstetrics and Gynecology, 98, 525.

[10] Melamed, N., Chen, R., Soiberman, U., Ben-Haroush, A., Hod, M. and Yogev, Y. (2008) Spontaneous and indicated preterm delivery in pregestational diabetes mellitus: Etiology and risk factors. Achieves of Gynecology and $\mathrm{Ob}$ stetrics, 278, 129-134.

[11] Lawrence, J.M., Contreras, R., Chen, W. and Sacks, D.A. (2008) Trends in the prevalence of pre existing diabetes and gestational diabetes mellitus among a racially/ethnically diverse population of pregnant women, 1999-2005. Diabetes Care, 31, 899-904. doi:10.2337/dc07-2345 